\title{
THE CASIMIR FORCE BETWEEN METALLIC MIRRORS
}

\author{
ASTRID LAMBRECHT, CYRIAQUE GENET AND SERGE REYNAUD \\ Laboratoire Kastler Brossel * \\ Université Pierre et Marie Curie, Ecole Normale Supérieure et CNRS \\ Campus Jussieu, Case 74, 75252 Paris Cedex 05, France
}

\begin{abstract}
In order to compare recent experimental results with theoretical predictions we study the influence of finite conductivity of metals on the Casimir effect. The correction to the Casimir force and energy due to imperfect reflection and finite temperature are evaluated for plane metallic plates where the dielectric functions of the metals are modeled by a plasma model. The results are compared with the common approximation where conductivity and thermal corrections are evaluated separately and simply multiplied.
\end{abstract}

\section{Introduction}

After its prediction in 1948 the Casimir force has been observed in a number

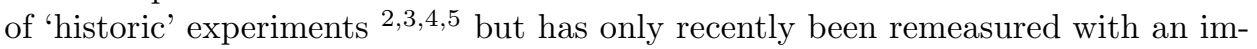
proved experimental precision 6 . An accurate comparison with the predictions of Quantum Field Theory should therefore now be possible, provided that theoretical predictions account for the differences between real experiments and the idealized Casimir situation. In particular, experiments are performed at room temperature between metallic mirrors while theoretical calculations are often performed at zero temperature and for perfect reflectors. As the experimental accuracy is claimed to be up to the order of $1 \%$, theoretical expectations should also be computed with the same accuracy if the aim is to test agreement between theory and experiment. A high accuracy is also important in order to control the effect of Casimir force when studying small short range forces 0.10 .11 .

The influence of thermal field fluctuations on the Casimir forfere known to become important for distances of the order of a typical length $22.13 .42,5$

$$
\lambda_{\mathrm{T}}=\frac{2 \pi c}{\omega_{\mathrm{T}}}=\frac{\hbar c}{k_{B} T}
$$

When evaluated at room temperature, $\lambda_{\mathrm{T}}$ amounts to approximately $7 \mu \mathrm{m}$. In contrast, the finite conductivity of metals has an appreciable effect for distances smaller than or of the order of the plasma wavelength $\lambda_{\mathrm{P}}$ determined by the plasma frequency $\omega_{\mathrm{P}}$ of the metal (see 16 and references therein)

$$
\lambda_{\mathrm{P}}=\frac{2 \pi c}{\omega_{\mathrm{P}}}
$$

For metals used in the recent experiments, this wavelength lies in the range $0.1 \mu \mathrm{m}$ $0.2 \mu \mathrm{m}$. This means that conductivity and thermal corrections to the Casimir force are important in quite different distance ranges.

The purpose of this contribution is to give an accurate evaluation of the Casimir force $F$ taking into account finite conductivity and temperature corrections at the

*mailto:lambrecht@spectro.jussieu.fr; http://www.spectro.jussieu.fr/Vacuum 
same time. To characterize the whole correction, we will compute the factor $\eta_{\mathrm{F}}$ describing the combined effect of conductivity and temperature

$$
\begin{aligned}
\eta_{\mathrm{F}} & =\frac{F}{F_{\mathrm{Cas}}} \\
F_{\mathrm{Cas}} & =\frac{\hbar c A \pi^{2}}{240 L^{4}}
\end{aligned}
$$

$F_{\text {Cas }}$ is the ideal Casimir force corresponding to perfect mirrors in vacuum. $L$ is the distance between the mirrors, $A$ their surface and $\hbar$ and $c$ respectively the Planck constant and the speed of light. We will also evaluate the factors associated with each effect taken separately from each other

$$
\eta_{\mathrm{F}}^{\mathrm{P}}=\frac{F^{\mathrm{P}}}{F_{\mathrm{Cas}}} \quad \eta_{\mathrm{F}}^{\mathrm{T}}=\frac{F^{\mathrm{T}}}{F_{\mathrm{Cas}}}
$$

$F^{\mathrm{P}}$ is the Casimir force evaluated by accounting for finite conductivity of the metals but assuming zero temperature and $F^{\mathrm{T}}$ is the Casimir force evaluated at temperature $T$ for perfect reflectors.

Now the question is to which level of accuracy the complete correction factor $\eta_{\mathrm{F}}$ can be approximated as the product of the factors $\eta_{\mathrm{F}}^{\mathrm{P}}$ and $\eta_{\mathrm{F}}^{\mathrm{T}}$ ? To answer this question we will evaluate the quantity

$$
\delta_{\mathrm{F}}=\frac{\eta_{\mathrm{F}}}{\eta_{\mathrm{F}}^{\mathrm{P}} \eta_{\mathrm{F}}^{\mathrm{T}}}-1
$$

which measures the degree of validity of the approximation where both effects are evaluated independently from each other. We will give an analytical estimation of this deviation in the end of this paper. We will also give the same results for the Casimir energy by defining a factor $\eta_{\mathrm{E}}$ measuring the whole correction of Casimir energy due to conductivity and temperature and then discussing the factors $\eta_{\mathrm{E}}^{\mathrm{P}}$ and $\eta_{\mathrm{E}}^{\mathrm{T}}$ and the deviation $\delta_{\mathrm{E}}$ in the same manner as for the force.

In view of a comparison between experimental and theoretical results, it has to be noted that recent experiments have not been performed in the plane-plane but in the plane-sphere configuration. The Casimir force in this geometry is usually estimated from the proximity theorem 17 $28,19,20,21$. Basically this amounts to evaluating the force by adding the contributions of various distances as if they were independent. In the plane-sphere geometry the force evaluated in this manner turns out to be given by the Casimir energy evaluated in the plane-plane configuration for the distance $L$ being defined as the distance of closest approach in the plane-sphere geometry. Hence, the factor $\eta_{\mathrm{E}}$ evaluated here for the energy can be used to infer the factor for the force measured in the plane-sphere geometry. Surface roughness corrections will not be considered in the following. Finally the dielectric response of the metallic mirrors will be described by a plasma model. This model is known to describe correctly the Casimir force in the long distance range which is relevant for the study of temperature effects. 


\section{Casimir force and free energy}

When real mirrors are characterized by frequency dependent reflection coefficients, the Casimir force is obtained as an integral over frequencies and wavevectors associated with vacuum and thermal fluctuations 22. The Casimir force is a sum of two parts corresponding to the 2 field polarizations with the two parts having the same form in terms of the corresponding reflection coefficients

$$
\begin{aligned}
& F=\sum_{k=-\infty}^{\infty} \frac{\omega_{\mathrm{T}}}{2} \mathcal{F}\left[k \omega_{\mathrm{T}}\right] \\
& \mathcal{F}[\omega \geq 0]=\frac{\hbar A}{2 \pi^{2}} \int_{\frac{\omega}{c}}^{+\infty} \mathrm{d} \kappa \kappa^{2} f \\
& f=\frac{r_{\perp}^{2}(i \omega, i \kappa)}{e^{2 \kappa L}-r_{\perp}^{2}(i \omega, i \kappa)}+\frac{r_{\|}^{2}(i \omega, i \kappa)}{e^{2 \kappa L}-r_{\|}^{2}(i \omega, i \kappa)} \\
& \mathcal{F}[-\omega]=\mathcal{F}[\omega]
\end{aligned}
$$

$r_{\perp}$ (respectively $r_{\|}$) denotes the amplitude reflection coefficient for the orthogonal (respectively parallel) polarization of one of the two mirrors. The mirrors are here supposed to be identical, otherwise $r_{\perp}^{2}$ should be replaced by the product of the two coefficients. $\omega$ is the frequency and $\kappa$ the wavevector along the longitudinal direction of the cavity formed by the 2 mirrors. $\mathcal{F}[\omega]$ is defined for positive frequencies and extended to negative ones by parity.

The Casimir force (6) may alse be rewritten after a Fourier transformation, as a consequence of Poisson formula 15

$$
\begin{aligned}
F & =\sum_{m=-\infty}^{\infty} \widetilde{\mathcal{F}}\left(m \lambda_{\mathrm{T}}\right) \\
\widetilde{\mathcal{F}}(x) & =\int_{0}^{\infty} \mathrm{d} \omega \cos \left(\frac{\omega x}{c}\right) \mathcal{F}[\omega]
\end{aligned}
$$

The contribution of vacuum fluctuations, that is also the limit of a null temperature $\left(\omega_{\mathrm{T}} \rightarrow 0\right)$ in (6), corresponds to the contribution $m=0$ in (7)

$$
F^{\mathrm{P}}=\tilde{\mathcal{F}}(0)=\int_{0}^{\infty} \mathrm{d} \omega \mathcal{F}[\omega]
$$

Hence, the whole force (7) is the sum of this vacuum contribution $m=0$ and of thermal contributions $m \neq 0$.

We will consider metallic mirrors with the dielectric function $\varepsilon(i \omega)$ for imaginary frequencies given by the plasma model

$$
\varepsilon(i \omega)=1+\frac{\omega_{\mathrm{P}}^{2}}{\omega^{2}}
$$

$\omega_{\mathrm{P}}$ is the plasma frequency related to the plasma wavelength $\lambda_{\mathrm{P}}$ by (2). For the metals used in recent experiments, the values chosen for the plasma wavelength $\lambda_{\mathrm{P}}$ will be $107 \mathrm{~nm}$ for $\mathrm{Al}$ and $136 \mathrm{~nm}$ for $\mathrm{Cu}$ and $\mathrm{Au}$.

MG9: submitted to World Scientific on January 12, 2001 
We will also focus our attention on mirrors with a large optical thickness for which the reflection coefficients $r_{\perp}(i \omega, i \kappa)$ and $r_{\|}(i \omega, i \kappa)$ correspond to a simple vacuum-metal interface. Their expressions can be found in standard literature.

The Casimir energy will be obtained from the force by integration over the mirrors relative distance

$$
E=\int_{L}^{\infty} F(x) \mathrm{d} x
$$

As this procedure is performed at constant temperature, the energy thus obtained corresponds to the thermodynamical definition of a free energy. For simplicity we will often use the denomination of an energy. We will define a factor $\eta_{\mathrm{E}}$ measuring the whole correction of energy due to conductivity and temperature effects with respect to the ideal Casimir energy

$$
\begin{aligned}
\eta_{\mathrm{E}} & =\frac{E}{E_{\mathrm{Cas}}} \\
E_{\text {Cas }} & =\frac{\hbar c A \pi^{2}}{720 L^{3}}
\end{aligned}
$$

The positive value of the energy here means that the Casimir energy is a binding energy while the positive value of the force is associated with an attractive character.

\section{Numerical evaluations}

In the following we present the numerical evaluation of the correction factors of the Casimir force and energy using equations written in the former section.

The force correction factor was evaluated for the experimentally relevant distance range of $0.1-10 \mu \mathrm{m}$ with the help of equation (7), supposing explicitly a plasma model for the dielectric function, and the result was normalized by the ideal Casimir force. The energy correction factor was then calculated by numerically integrating the force and normalizing by the ideal Casimir energy. Integration was restricted to a finite interval, the upper limit exceeding at least by a factor of $10^{4}$ the distance at which the energy value was calculated. Extending the integration range by a factor of 100 changed the numerical result by less than $10^{-7}$.

The results of the numerical evaluation of $\eta_{\mathrm{F}}$ are shown as the solid lines in figures 1 for $\mathrm{Al}$ and for $\mathrm{Cu}-\mathrm{Au}$ assuming a temperature of $T=300 \mathrm{~K}$. They are compared with the force reduction factor $\eta_{\mathrm{F}}^{\mathrm{P}}$ due to finite conductivity (dashed lines) and the force enhancement factor $\eta_{\mathrm{F}}^{\mathrm{T}}$ calculated for perfect mirrors at $300 \mathrm{~K}$ (dashed-dotted lines).

Figure 2 shows similar results for the factor $\eta_{\mathrm{E}}$ obtained through numerical evaluation of the Casimir free energy. The shape of the graphs is similar to the ones of the force. However, while finite conductivity corrections are more important for the force, thermal effects have a larger influence on energy.

For the force as well as for the energy, temperature corrections are negligible in the short distance limit while conductivity corrections may be ignored at large distances. The whole correction factor $\eta$ behaves roughly as the product $\eta^{\mathrm{P}} \eta^{\mathrm{T}}$ of the 2 correction factors evaluated separately. However, both correction factors are appreciable in the distance range $1-4 \mu \mathrm{m}$ in between the two limiting cases.

MG9: submitted to World Scientific on January 12, 2001 

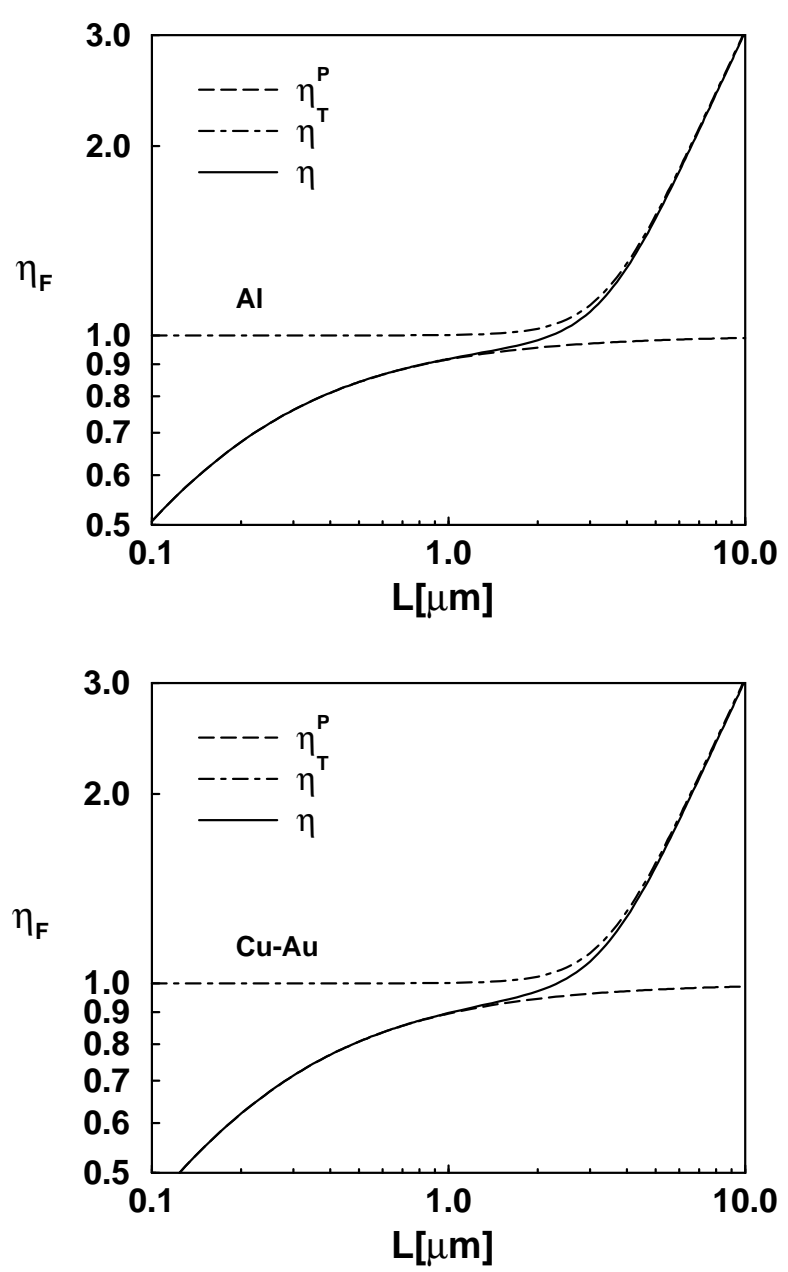

Figure 1. Force correction factor for $\mathrm{Al}$ (upper figure) and $\mathrm{Cu}$ and $\mathrm{Au}$ (lower graph) as function of the mirrors distance at $T=300 \mathrm{~K}$.

Since this range is important for the comparison between experiments and theory, it is necessary to discuss more precisely how good is the often used approximation which identifies $\eta$ to the product $\eta^{\mathrm{P}} \eta^{\mathrm{T}}$. In order to assess the quality of this approximation, we have plotted in figure 3 the quantities $\delta_{\mathrm{F}}$ and $\delta_{\mathrm{E}}$ (cf. eq.(5) as a function of the distance for $\mathrm{Al}, \mathrm{Cu}-\mathrm{Au}$ and two additional plasma wavelengths. A value of $\delta=0$ would signify that the approximation gives an exact estimation of the whole correction. An important outcome of our calculation is that the errors $\delta_{\mathrm{F}}$ and $\delta_{\mathrm{E}}$ are of the order of $1 \%$ for $\mathrm{Al}$ and $\mathrm{Cu}-\mathrm{Au}$ at a temperature of $300 \mathrm{~K}$.

For estimations at the $5 \%$ level, the separate calculation of $\eta^{\mathrm{P}}$ and $\eta^{\mathrm{T}}$ and the evaluation of $\eta$ as the product $\eta^{\mathrm{P}} \eta^{\mathrm{T}}$ can therefore be used. However, if a $1 \%$ level 

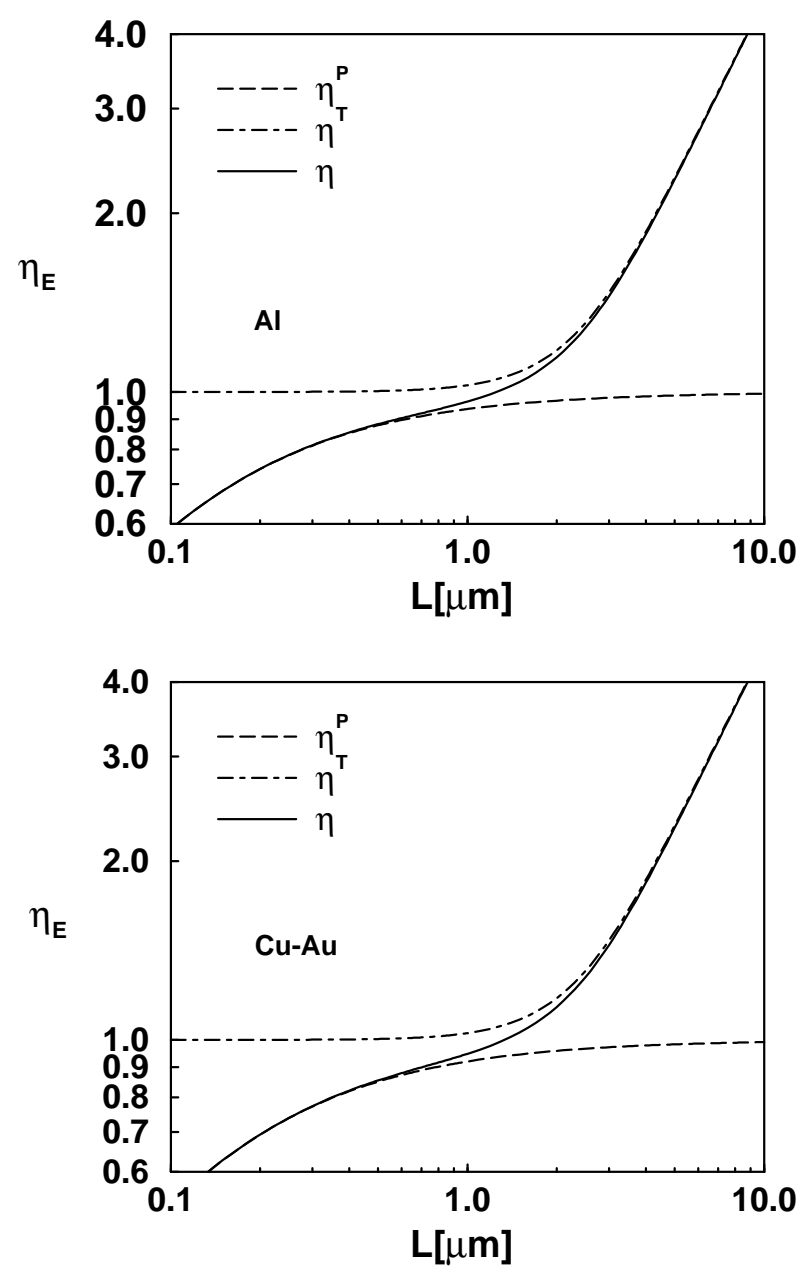

Figure 2. Energy correction factor for $\mathrm{Al}$ (upper figure) and $\mathrm{Cu}$ and $\mathrm{Au}$ (lower graph) as function of the mirrors distance at $T=300 \mathrm{~K}$.

or a better accuracy is aimed at, this approximation is not sufficient. It should be noticed furthermore that the error increases when the temperature or the plasma wavelength are increased. It becomes of the order of $4 \%$ for a plasma wavelength of $0.5 \mu \mathrm{m}$ at $300 \mathrm{~K}$. The sign obtained for $\delta$ means that the approximation gives too small values of force and energy.

\section{Scaling laws for the deviations}

An inspection of figure 3 shows that the curves corresponding to different plasma wavelengths $\lambda_{\mathrm{P}}$ have similar shapes with a maximum which is practically attained 

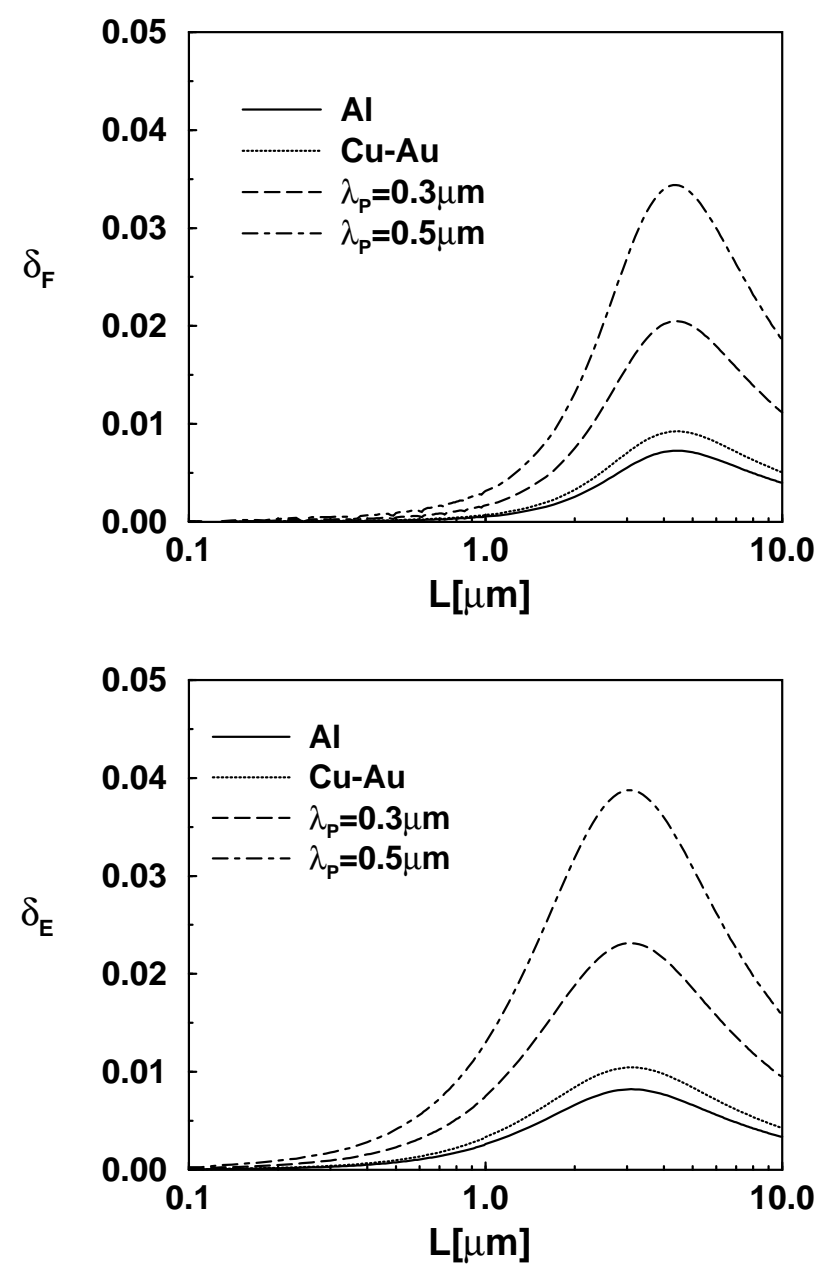

Figure 3. $\delta_{\mathrm{F}}$ (upper graph) and $\delta_{\mathrm{E}}$ (lower graph) as a function of the mirrors distance. The results are given for the three metals $\mathrm{Al}, \mathrm{Cu}-\mathrm{Au}$ and two larger plasma wavelengths.

for the same distance between the mirrors. The amplitude of the deviations, which is larger for the energy than for the force, is found to vary linearly as a function of the plasma wavelength $\lambda_{\mathrm{P}}$.

This scaling property is confirmed by figure 1 where we have drawn the deviations after an appropriate rescaling

$$
\Delta=\frac{\lambda_{\mathrm{T}}}{\lambda_{\mathrm{P}}} \delta
$$

The curves obtained for $\Delta_{\mathrm{F}}$ and $\Delta_{\mathrm{E}}$ for different plasma wavelengths at temperature $T=300 K$ are nearly perfectly identical to each other. These curves correspond to values of the plasma wavelength small compared to the thermal wavelength and 
the scaling law would not be obeyed so well otherwise.
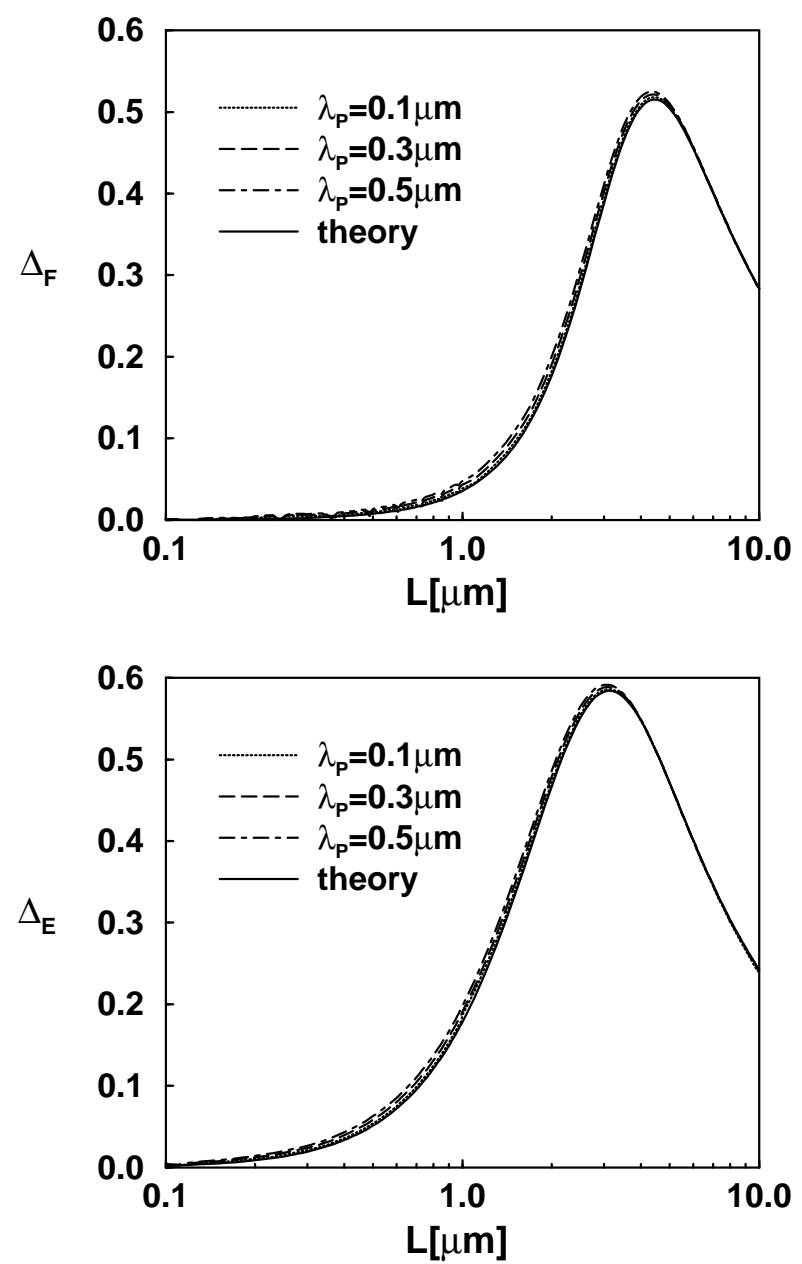

Figure 4. The deviations are represented for the force (upper graph) and the free energy (lower graph) after the rescaling described by equation (12). Different plasma wavelengths lead to nearly identical functions, drawn as dotted, dashed and dotted-dashed lines. These functions are hardly distinguishable from the solid lines which represent the analytical expressions derived in the next section.

In other words, the deviations $\delta_{\mathrm{F}}$ and $\delta_{\mathrm{E}}$ are proportional to the factor $\frac{\lambda_{\mathrm{P}}}{\lambda_{\mathrm{T}}}$ on one hand, and to the functions $\Delta_{\mathrm{F}}$ and $\Delta_{\mathrm{E}}$ on the other hand. The latter functions, which no longer depend on $\lambda_{\mathrm{P}}$, provide a simple method for reaching a good accuracy in the theoretical estimation of the whole correction factor

$$
\eta=\eta^{\mathrm{P}} \eta^{\mathrm{T}}\left(1+\frac{\lambda_{\mathrm{P}}}{\lambda_{\mathrm{T}}} \Delta\right)
$$


This method is less direct than the complete numerical integration of the forces which has been performed for obtaining the curves presented in the previous section. But it requires easier computations while nevertheless giving accurate estimations of the correction factors. Typically, the deviation $\delta$ with a magnitude of the order of the $\%$ may be estimated with a much better precision through the mere inspection of figure 4 .

One may explain this scaling law by using a partial analytical integration of the whole correction factors and calculating analytical expressions for the functions $\Delta_{F}$ and $\Delta_{\mathrm{E}}$ to first order in $\lambda_{\mathrm{P}}$ :

$$
\begin{aligned}
& \Delta_{\mathrm{F}}=\frac{8}{3 \pi} \frac{\lambda_{T}}{L} \frac{\eta_{\mathrm{F}}^{\mathrm{T}}-1}{\eta_{\mathrm{F}}^{\mathrm{T}}}+\frac{\lambda_{T}}{L} \frac{\phi_{\mathrm{F}}}{\eta_{\mathrm{F}}^{\mathrm{T}}} \\
& \Delta_{\mathrm{E}}=\frac{2}{\pi} \frac{\lambda_{T}}{L} \frac{\eta_{\mathrm{E}}^{\mathrm{T}}-1}{\eta_{\mathrm{E}}^{\mathrm{T}}}+\frac{\lambda_{T}}{L} \frac{\phi_{\mathrm{E}}}{\eta_{\mathrm{E}}^{\mathrm{T}}}
\end{aligned}
$$

This function is plotted as the solid line on figure 1 and it is found to fit well the results of the complete numerical integration presented before. The detailed calculations may be found in 23 .

\section{Summary}

We have given an accurate evaluation of the Casimir force and Casimir free energy between 2 plane metallic mirrors, taking into account conductivity and temperature corrections at the same time. The whole corrections with respect to the ideal Casimir formulas, corresponding to perfect mirrors in vacuum, have been characterized by factors $\eta_{\mathrm{F}}$ for the force and $\eta_{\mathrm{E}}$ for the energy. These factors have been computed through a numerical evaluation of the integral formulas. They have also been given a simplified form as a product of 3 terms, namely the reduction factor associated with conductivity at null temperature, the increase factor associated with temperature for perfect mirrors, and a further deviation factor measuring a kind of interplay between the two effects. This last factor turns out to lie in the $1 \%$ range for metals used in the recent experiments performed at ambient temperature. Hence the conductivity and temperature corrections may be treated independently from each other and simply multiplied for theoretical estimations above this accuracy level.

However, when accurate comparisons between experimental and theoretical values of the Casimir force are aimed at, the deviation factor has to be taken into account in theoretical estimations. The deviation factor is appreciable for distances greater than the plasma wavelength $\lambda_{\mathrm{P}}$ but smaller or of the order of the thermal wavelength $\lambda_{\mathrm{T}}$. We have used this property to derive a scaling law of the deviation factor. This law allows one to obtain a simple but accurate estimation of the Casimir force and free energy through a mere inspection of figure 4 . Alternatively one can use analytical expressions which have been obtained through a first order expansion in $\lambda_{\mathrm{P}}$ of the thermal contributions to Casimir forces and fit well the results of complete numerical integration.

We have represented the optical properties of metals by the plasma model. This model does not lead to reliable estimations of the forces at small distances but this 
deficiency may be corrected by using the real dielectric function of the metals. This does not affect the discussion of the present paper, except for the fact that the pure conductivity effect has to be computed through an integration of optical data for distances smaller than $0.5 \mu \mathrm{m}$. Finally surface roughness corrections, which have not been considered in the present paper, are expected to play a significant role in theory-experiment comparisons in the short distance range.

\section{References}

1. H.B.G. Casimir, Proc. Kon. Nederl. Akad. Wet. 51, 793 (1948)

2. B.V. Deriagin and I.I. Abrikosova, Soviet Physics JETP 3, 819 (1957)

3. M.J. Sparnaay, Physica XXIV, 751 (1958); W. Black, J.G.V. De Jongh, J.Th.G. Overbeek and M.J. Sparnaay, Transactions of the Faraday Society 56, 1597 (1960)

4. D. Tabor and R.H.S. Winterton, Nature 219, 1120 (1968)

5. E.S. Sabisky and C.H. Anderson, Phys. Rev. A 7, 790 (1973)

6. S.K. Lamoreaux, Phys. Rev. Lett. 78, 5 (1997); erratum in Phys. Rev. Lett. 81, $5475(1998)$

7. U. Mohideen and A. Roy, Phys. Rev. Lett. 81, 4549 (1998)

8. A. Roy, C. Lin and U. Mohideen, Phys. Rev. D 60, 111101 (1999)

9. E. Fischbach and C. Talmadge, in The Search for Non Newtonian Gravity (AIP Press/Springer Verlag, 1998) and references therein; E. Fischbach and D.E. Krause, Phys. Rev. Lett. 82, 4753 (1999)

10. G. Carugno, Z. Fontana, R. Onofrio and C. Rizzo, Phys. Rev. D 55, 6591 (1997)

11. M. Bordag, B. Geyer, G.L. Klimchitskaya, and V.M. Mostepanenko, Phys. Rev. D 60, 055004 (1999)

12. E.M. Lifshitz, Sov. Phys. JETP 273 (1956); E.M. Lifshitz and L.P. Pitaevskii, Landau and Lifshitz Course of Theoretical Physics: Statistical Physics Part 2 ch VIII (Butterworth-Heinemann, 1980)

13. J. Mehra, Physica 57, 147 (1967)

14. L.S. Brown and G.J. Maclay, Phys. Rev. 184, 1272 (1969)

15. J. Schwinger, L.L. de Raad Jr., and K.A. Milton, Annals of Physics 115, 1 (1978)

16. A. Lambrecht and S. Reynaud, Eur. Phys. J. D 8, 309 (2000)

17. B.V. Deriagin, I.I. Abrikosova and E.M. Lifshitz, Quart. Rev. 10, 295 (1968)

18. J. Blocki, J. Randrup, W.J. Swiatecki and C.F. Tsang, Ann. Physics 105, 427 (1977)

19. V.M. Mostepanenko and N.N. Trunov Sov. J. Nucl. Phys. 42, 812 (1985)

20. V.B. Bezerra, G.L. Klimchitskaya and C. Romero, Mod. Phys. Lett. 12, 2613 (1997)

21. G.L. Klimchitskaya, A. Roy, U. Mohideen and V.M. Mostepanenko, Phys. Rev. A 60, 3487 (1999)

22. M.T. Jaekel and S. Reynaud, J. Physique I-1, 1395 (1991)

23. C. Genet, A. Lambrecht, and S. Reynaud, Phys. Rev. A 62, 012110 (2000) 